\title{
A systematic review and meta-analysis of topoisomerase inhibition in pre-clinical glioma models
}

\author{
Toni Rose Jue ${ }^{1}$, Emily S. Sena ${ }^{2}$, Malcolm R. Macleod ${ }^{2}$, Kerrie L. McDonald ${ }^{1}$ and \\ Theodore C. Hirst ${ }^{2,3}$ \\ ${ }^{1}$ Cure Brain Cancer Neuro-Oncology Group, Prince of Wales Clinical School, UNSW Sydney, Australia \\ ${ }^{2}$ Center for Clinical Brain Sciences, University of Edinburgh, Edinburgh, UK \\ ${ }^{3}$ Department of Neurosurgery, Royal Victoria Hospital, Belfast, UK \\ Correspondence to: Theodore C. Hirst, email: Theodore.Hirst@ed.ac.uk \\ Keywords: topoisomerase; glioma; systematic review; meta-analysis; meta-regression \\ Received: September 04, $2017 \quad$ Accepted: January 20, $2018 \quad$ Published: January 29, 2018 \\ Copyright: Jue et al. This is an open-access article distributed under the terms of the Creative Commons Attribution License 3.0 \\ (CC BY 3.0), which permits unrestricted use, distribution, and reproduction in any medium, provided the original author and source \\ are credited.
}

\section{ABSTRACT}

Malignant glioma is a devastating disease affecting both adults and children with limited treatment strategies. Pre-clinical animal studies are critical to the development and planning of novel treatment designs for human clinical trials. Topoisomerases has been a target of interest in the treatment of high grade gliomas, such as glioblastoma, in the past years. Here we assess pre-clinical glioma literature with the aim to identify predictive variables that favour treatment outcomes from topoisomerase inhibition. Data was extracted from 90 experimental comparisons, this was divided based on available survival $(n=61)$ and tumor volume $(n=29)$ data. The meta-analysis revealed that the overall effect of topoisomerase inhibition prolonged survival by a factor of 1.33 (95\% CI: 1.23-1.43) and reduced tumor growth by a factor of 3.21 (95\% CI: 1.99-5.88), with considerable between-study heterogeneity. Multivariable meta-regression identified glioma model, type of control, route of drug administration and drug of choice to be predictive of improved survival outcome. Publication bias assessment by contour-enhanced funnel plots, Egger's regression test and trim and fill analysis showed evidence of publication bias in all studies. This study identified multiple study design factors that should be taken into consideration to improve the translation of pre-clinical investigation of topoisomerase inhibition into clinical use.

\section{INTRODUCTION}

Glioblastoma (GBM) is the most common malignant brain tumor with an incidence rate of 3.20 per 100,000 population [1]. GBM increases in incidence from the age of 30 years and reaching its peak at 75 to 84 years old, with a median age of 65 years old [1-5]. The addition of temozolomide (TMZ) to surgical resection and radiotherapy represented the last significant advance in GBM management [6]. However, median survival remains at less than 15 months and TMZ resistance has since been reported in a proportion of tumors in which the
$\mathrm{O}^{6}$-methylguanine methyltransferase (MGMT) promoter is not methylated [7-11]. In the past 40 years, only a handful of drugs have been approved for the treatment of GBM in the USA, including temozolomide (2005), bevacizumab (2009), carmustine (1996) and lomustine (1976). Overall there have been no major advances in chemotherapy for GBM in the last decade and, consequently, there is an urgent need for new treatments.

Pre-clinical animal studies form an important stage in the development and planning of new treatment designs for human clinical trials. Systematic review and meta-analysis is a powerful tool for the assessment of any treatment and 
is considered to be the highest level of clinical evidence by many regulatory bodies. Although systematic review and meta-analyses of clinical trials are widely reported, until recently there has been a relative paucity concerning pre-clinical literature. Groups such as the Collaborative Approach to Meta-Analysis and Review of Animal Data from Experimental Studies (CAMARADES) have undertaken systematic review and meta-analysis across a range of pre-clinical disease groups which have illustrated several important themes relevant to the design and interpretation of animal experiments $[12,13]$.

Previous systematic reviews of glioma model treatments have assessed the efficacy of BCNU/CCNU [14], temozolomide [15] and gene therapies [16]. In these reviews, particularly in relation to chemotherapies, results were overall concordant with human trials, although the underlying data were limited in quality and design. Several factors were found to be associated with observed treatment efficacy including factors relating to the reporting of measures to address potential risks of bias such as randomization and blinding; and those relating to study design, in particular the tumor models used.

Tumor Suppressor Protein 53 (TP53) is highly associated with the regulation of topoisomerase I and II activity [17-19]. Mutations in the TP53 gene has been found to upregulate topoisomerase II expression while leaving topoisomerase I expression unaffected $[18,20]$. Topoisomerase I and II enzymes are essential in the uncoiling of supercoiled DNA to promote DNA metabolic processes. Topoisomerase I catalyse singlestrand breaks that allows DNA transcription needed for protein synthesis, while Topoisomerase II catalyses the double strand breaks important for chromosome condensation. Inhibition of topoisomerases lead to the increased formation of stable topoisomerase-cleaved DNA breaks which eventually lead to cell death [21, 22]. Topoisomerase inhibition is an established strategy in oncology, and has been a target of interest in a range of different cancer types including glioma. TP53 mutations are present in the majority of primary GBMs and may contribute to tumorigenesis [23-25]. In the last 5 years, Phase I and II clinical trials evaluating the use of topoisomerase inhibitors in combination with other chemotherapeutic drugs to treat GBM have shown variable results [26-33]. In 2013, a meta-analysis performed by Leonard and Wolff [34] of data from phase I-III clinical trials from 1976 to 2011 (including 44,850 patients from 624 publications) examined the treatment efficacy of four topoisomerase inhibitors (topotecan, irinotecan, etoposide and teniposide). Of these, etoposide appeared to confer the greatest advantage in terms of increase in median survival, while others showed either no significant improvement or worsened outcome.

This variability in effects observed in clinical trials suggests that topoisomerase inhibition may have a role in treatment of glioma if the optimal conditions for efficacy (patient selection, timing of treatment, etc.) could be identified. This contrasts with the pre-clinical literature where the subjective impression is one of consistent efficacy. Here we describe a systematic review and metaanalysis of topoisomerase inhibition in pre-clinical GBM studies, with the aim of summarising the overall efficacy of topoisomerase inhibition in this population and whether differences in efficacy exist between different drugs and different tumour models. Furthermore, we appraise the quality of these studies and search for evidence of bias; as well as describing the variation in and impact of different study design parameters on outcome. Finally, we aim to appraise the construct validity of the models used.

\section{RESULTS}

After searching PubMed, Medline and EMBASE, 547 publications were identified with 76 publications satisfying the inclusion criteria (Figure 1). We excluded one study that used bioluminescent imaging to assess for change in tumor volume, as there were no direct measurements of tumor volume [35]. Other reasons for exclusion were missing or unreported data $(n=5)$, no appropriate control used for the study $(n=11)$, no measure of the variance of the volume data $(n=2)$ or the tumor volumes not reported $(n=2)$, no quantitative data reported for the outcome measure $(n=1)$, no individual drug treatment results reported $(n=2)$ and the drug being assessed in the study is not included in the list of drugs specified in the protocol $(n=1)$.

Following these exclusions, 52 publications remained reporting 61 experimental comparisons assessing animal median survival (MSR) and 29 assessing tumor volume (TVR) data; using a total of 1,611 animals. The details of the included studies are presented in Supplementary Table 1.

Of the 90 comparisons, mice were the most commonly used species for assessing both survival ( $n$ $=35$ ) and tumor volume reduction $(n=20)$. Mice and rats were the only species of animal used. Comorbid animals were commonly used. Athymic animal models were most commonly observed in studies assessing survival $(n=26)$ and in studies assessing tumor volume $(n=20)$. Other comorbidities observed in the survival studies were RAG2-M $(n=2)$ and SCID $(n=2)$, which are both immunocompromised models. SCID animals were reported in two out of 29 tumor volume reduction experimental comparisons. Survival studies included 56 intracranial and five subcutaneous animal models, while tumor volume reduction studies included 11 intracranial and 18 subcutaneous animal models.

The most frequently used cell line was U87 in both survival $(n=14)$ and volume studies $(n=16)$. Other glioma models more commonly observed were U251 $(n=7), 9 \mathrm{~L}(n=8), \mathrm{BT} 4 \mathrm{Ca}(n=5), \mathrm{GBM}(n=7)$, and 101/8 $(n=6)$. Aside from one study which used a patient-derived 
GBM xenograft model [36], none of the studies screened for TP53 mutation (Table 1). The majority of survival studies used xenograft models $(n=57)$, and six of the seven experiments that used 'GBM' models were serially passaged subcutaneous patient-derived xenografts. Most tumor models developed were orthotopic $(n=67)$.

Experiments most commonly used doxorubicin $(n=49)$, followed by irinotecan $(n=25)$, etoposide $(n=7)$, topotecan $(n=6)$, and epirubicin $(n=3)$. The most frequently used route of drug delivery were intravenous $(n=48)$, intracranial $(n=21)$ and intraperitoneal $(n=15)$ administration.

\section{Study quality}

Study quality was assessed in all 90 comparisons included using a predefined 12-item checklist (Supplementary Table 2). The study quality was modest, the median number of checklist items scored 6 out of 12 (IQR - 5-7, range 3-9). Ninety-six percent of the comparisons included in the systematic review were published in a peer-reviewed journal, 56\% reported random allocation to treatment groups, $10 \%$ blinded the assessment of outcome measures, none reported sample size calculation, $88 \%$ reported compliance with animal welfare regulations, $34 \%$ stated a potential conflict of interest, 26\% published "take rates", 13\% reported reasons for exclusions, $98 \%$ had a consistent site of tumor implantation, $87 \%$ reported a standard number of implanted cells in all the animals, $23 \%$ justified drug action and $62 \%$ justified the use of carriers.

\section{Meta-analysis}

In the 90 comparisons included in the systematic review, 18 different glioma models were reported

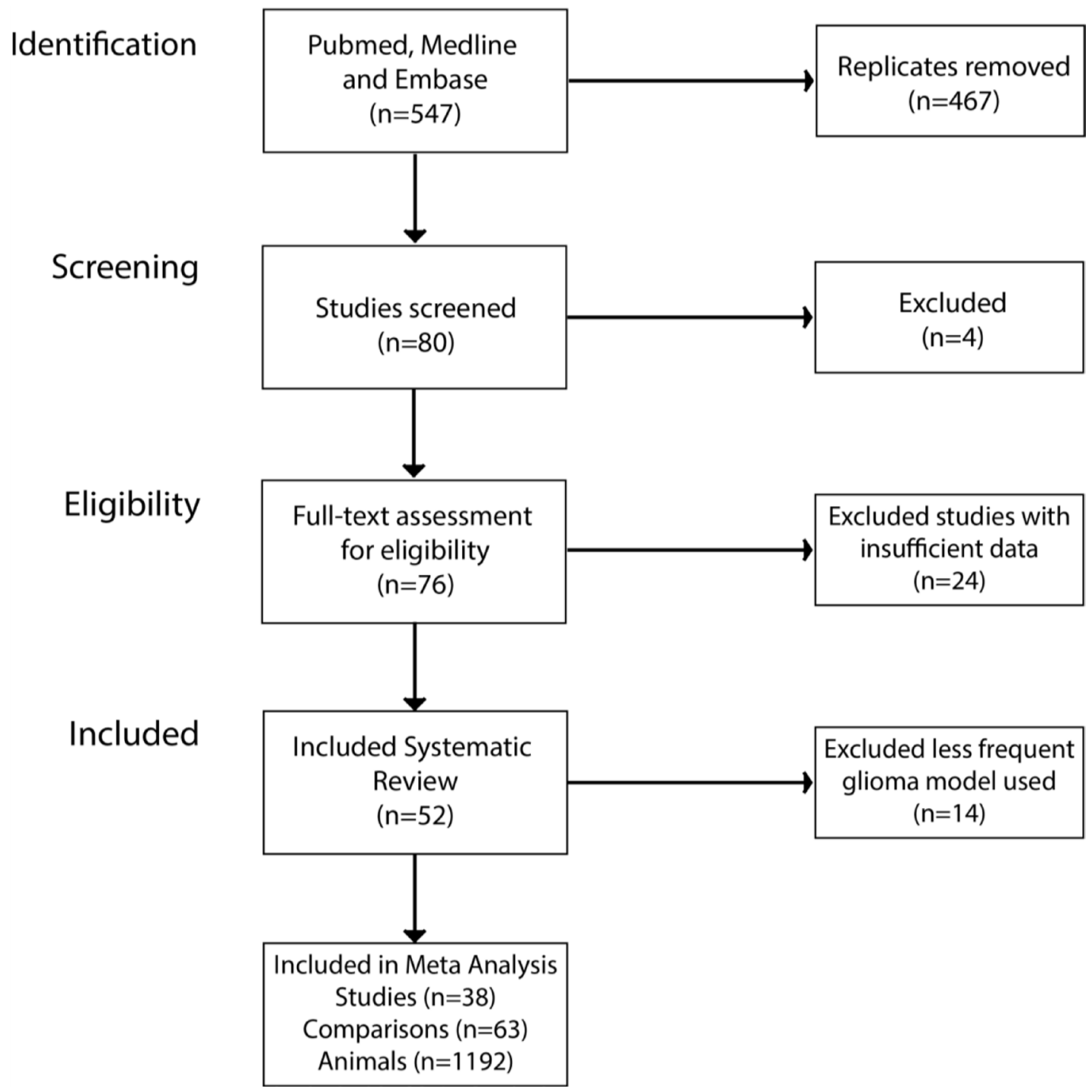

Figure 1: Study selection summary. Three public databases were searched for keyword of interest. This returned 547 publications, after screening using our inclusion criteria we were left with 38 publications included in the meta-analysis. 
Table 1: TP53 mutation status of glioma models

\begin{tabular}{lc}
\hline Cell line group & TP53 mutation status \\
\hline U87 & Wildtype [52] \\
U251 & Mutant [53] \\
GBM & SJ - GBM2 - mutant [36] \\
BT4Ca & unknown \\
9L & Mutant [54] \\
101/8 & unknown \\
\hline
\end{tabular}

Summary of TP53 mutation status for glioma models included in this meta-analysis.

(Supplementary Table 1). However, previous metaanalyses in this field have suggested a substantial degree of covariance between study design parameters, particularly the choice of tumor model [15, 16]. To limit this covariance, we proceeded to meta-analyse studies reporting experiments using glioma models which had been used in five or more studies reporting the corresponding outcome measure. This involved 47 survival and 16 tumor volume experimental comparisons using a total of 1,192 animals (Figure 2).

Overall, animals bearing gliomas treated with a topoisomerase inhibitor survived 1.32 times (95\% CI: 1.23 - 1.43) longer than control animals, substantial betweenstudy heterogeneity was observed $\left(F=7.1, p<0.001, I^{2}\right.$ $=92.2 \%)$. On the other hand, topoisomerase inhibition reduced tumor growth by $69 \%$ (TVR response ratio: 3.2; 95\% CI: 1.99 - 5.18) compared to control animals, substantial between-study heterogeneity was also observed in this dataset $\left(F=4.78, p<0.001, I^{2}=95.5 \%\right)$.

\section{Meta-regression}

Heterogeneity was high in both datasets, warranting further analysis to identify study quality or design items associated with treatment efficacy. As specified in our protocol, we used a multivariable meta-regression, designed to identify and account for covariance between study characteristics which is common in heterogeneous datasets. In this instance, we suspected glioma model selection to be a frequent confounder in univariable analyses.

We initially performed univariable meta-regression to assess the impact of study characteristics on both survival and tumor volume. Variables tested included the glioma model, animal species, site of tumor cell implantation, drug used, dosage, type of control used, route of drug delivery, frequency of drug administration, randomisation, blinding of outcome assessment and quality score. We deemed the data sufficient to proceed to multivariable meta-regression with the survival dataset (47 comparisons) but not the tumor volume dataset (18 comparisons).

For the multivariable meta-regression model, based on our previous experience, we allowed inclusion of one variable for every ten comparisons - thereby permitting input of five predictors. We selected these variables $a$ priori to be glioma model, site of implantation, drug used, route of delivery, quality score and type of control used for the experiment. However, because majority of the tumors were orthotopic $(n=44 / 47)$ we did not include site of implantation, instead we included the variable which had returned the largest $F$-value in the univariable analysis, that being the nature of the control used. Following the meta-regression, each predictor was tested post-hoc with a Wald test - with a significant result implying an independent predictive value of the variable of interest.

Our analysis of interest was the multivariable model; we report these data first. Where this was not possible, for remaining predictors in survival dataset and for the tumor volume dataset, we report results from the univariable meta-regression.

\section{Survival dataset}

The multivariable meta-regression model was significantly associated with median survival outcome, suggesting a predictive value of at least one variable offered $(F=6.08, p<0.0001)$, and residual $I^{2}$ of $74.24 \%$. When tested with a Wald test, 4 of 5 variables offered to the model (glioma model, drug, type of control, and route of drug delivery) were independently associated with heterogeneity in the survival dataset (Table 2).

All 4 drugs used appeared to be associated with treatment efficacy (Figure 3A), with the most commonly used drugs (doxorubicin and irinotecan) associated with similar outcomes (MSR: 1.29, 95\% CI 1.17-1.43 and $1.38,1.16-1.65$, respectively). Epirubicin, used in only 2 experiments, was associated with greater efficacy (1.78, $95 \%$ CI 1.19-2.68). The choice of drug was independently predictive of treatment outcome in the multivariable model $(F=3.74, p<0.05)$, although not in the univariable model $(F=1.14, p>0.0042)$. The route of drug delivery was associated with survival outcome in the multivariable model $(F=8.61, p<0.05$, Figure 3B), with intracranial treatment appearing to be associated with greater efficacy than systemic routes. There was no association seen on univariable analysis $(F=3.92, p>0.0042)$. Drug dose for doxorubicin $(F=0.01, p>0.0042)$ and irinotecan $(F$ 


\begin{tabular}{|c|c|c|c|}
\hline Author & Year & $\mathbf{N}$ & ES $[95 \% \mathrm{Cl}]$ \\
\hline $\begin{array}{l}\text { Wang } \\
\text { Marrero } \\
\text { Zhong } \\
\text { Tarasenko } \\
\text { Yang } \\
\text { Serwer } \\
\text { Serwer } \\
\text { Serwer } \\
\text { Guo } \\
\text { Vinchon-Petit } \\
\text { Kuroda } \\
\text { Kuroda } \\
\text { Kuroda } \\
\text { Kreuter } \\
\text { Petri } \\
\text { Ambruosi } \\
\text { Gomez-Manzano } \\
\text { Gomez-Manzano } \\
\text { Lesniak } \\
\text { Lesniak } \\
\text { Steiniger } \\
\text { Steiniger } \\
\text { Steiniger } \\
\text { Sharma } \\
\text { Sharma } \\
\text { Sharma } \\
\text { Glage } \\
\text { Glage } \\
\text { Glage } \\
\text { Verreault } \\
\text { Verreault } \\
\text { Baltes } \\
\text { Baltes } \\
\text { Recinos } \\
\text { Hsu } \\
\text { Chen } \\
\text { Chen } \\
\text { Chen } \\
\text { Chen } \\
\text { Chen } \\
\text { Li } \\
\text { Zhang } \\
\text { Verreault } \\
\text { Verreault } \\
\text { Zhao } \\
\text { Byeon } \\
\text { Ramachandran }\end{array}$ & $\begin{array}{l}2015 \\
2014 \\
2014 \\
2014 \\
2013 \\
2011 \\
2011 \\
2011 \\
2011 \\
2010 \\
2010 \\
2010 \\
2009 \\
2008 \\
2007 \\
2006 \\
2006 \\
2006 \\
2005 \\
2005 \\
2004 \\
2004 \\
2004 \\
1997 \\
1997 \\
1997 \\
2011 \\
2011 \\
2011 \\
2012 \\
2012 \\
2010 \\
2010 \\
2010 \\
2005 \\
2013 \\
2013 \\
2013 \\
2013 \\
2013 \\
2015 \\
2015 \\
2015 \\
2015 \\
2016 \\
2016 \\
2016\end{array}$ & $\begin{array}{r}6 \\
12 \\
9 \\
8.4 \\
9.3 \\
15 \\
12 \\
9.5 \\
7 \\
11.8 \\
5.7 \\
5.7 \\
9 \\
7.5 \\
23.3 \\
11.7 \\
36 \\
58 \\
15 \\
15 \\
27.2 \\
25.6 \\
10.5 \\
11 \\
9 \\
9.5 \\
16 \\
17 \\
18 \\
7.2 \\
7.2 \\
34 \\
25 \\
8 \\
20.3 \\
10.8 \\
10.8 \\
10.8 \\
10.8 \\
8.8 \\
16 \\
7.2 \\
7.2 \\
7.5 \\
8 \\
10.7 \\
26.7\end{array}$ & $\begin{array}{l}1.04[0.75-1.45] \\
1.02[0.87-1.20] \\
1.50[1.21-1.86] \\
0.84[0.67-1.06] \\
1.03[0.83-1.27] \\
1.27[1.11-1.44] \\
1.18[1.00-1.39] \\
1.06[0.86-1.31 \\
1.13[0.85-1.49] \\
1.12[0.95-1.32] \\
1.24[0.88-1.75] \\
1.17[0.83-1.65] \\
1.63[1.31-2.02 \\
1.80[1.39-2.34] \\
1.62[1.49-1.76] \\
1.25[1.06-1.48] \\
1.15[1.09-1.21] \\
1.20[1.16-1.24] \\
1.62[1.42-1.85] \\
2.14[1.88-2.44] \\
1.54[1.43-1.65] \\
1.85[1.71-1.99] \\
1.81[1.51-2.19] \\
0.94[0.79-1.12 \\
0.96[0.77-1.19] \\
1.02[0.83-1.25] \\
1.00[0.88-1.13] \\
1.18[1.05-1.32] \\
1.24[1.11-1.38] \\
1.16[0.89-1.53] \\
1.20[0.92-1.58] \\
1.31[1.24-1.39] \\
1.31[1.21-1.42] \\
2.38[1.87-3.05 \\
1.67[1.51-1.84] \\
1.42[1.19-1.70] \\
1.21[1.01-1.45 \\
1.79[1.49-2.15] \\
1.89[1.58-2.27] \\
3.16[2.53-3.95] \\
1.03[0.91-1.16] \\
1.31[1.00-1.73] \\
1.68[1.28-2.20] \\
1.68[1.29-2.18] \\
1.04[0.82-1.33] \\
1.07[0.89-1.29] \\
0.95[0.88-1.02]\end{array}$ \\
\hline Global & & 47 & $1.33[1.23-1.43]$ \\
\hline
\end{tabular}

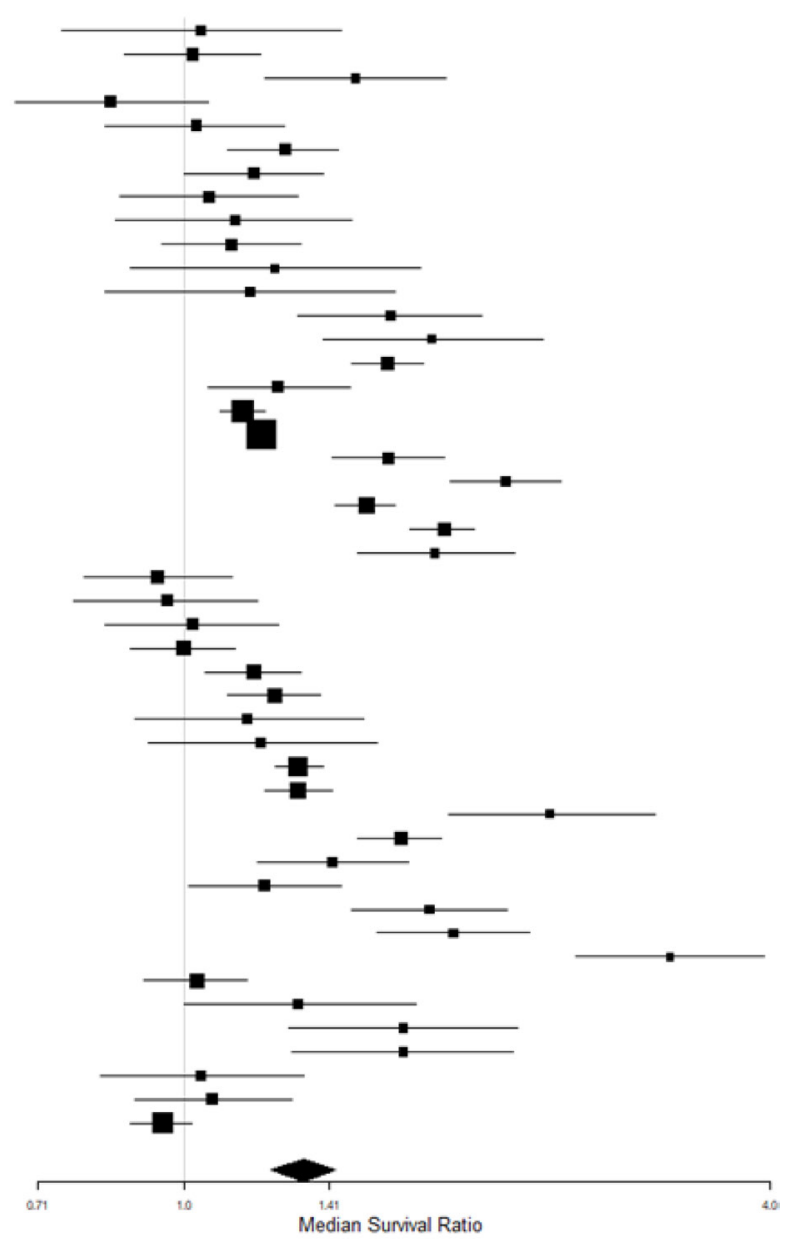

$\begin{array}{lrrr}\text { B Author } & \text { Year } & \text { N } & \text { ES [95\% Cl] } \\ \text { Zhong } & 2014 & 3 & 11.01[8.18-14.83] \\ \text { Jiang } & 2014 & 1.5 & 1.28[0.64-2.55] \\ \text { Jiang } & 2014 & 1.5 & 14.72[7.75-27.94] \\ \text { Escoffre } & 2013 & 2.5 & 2.27[0.65-7.95] \\ \text { Jaszberenyi } & 2013 & 2.5 & 2.22[0.87-5.65] \\ \text { Panigrahy } & 2010 & 1.7 & 2.12[1.41-3.19] \\ \text { Arai } & 2009 & 1.7 & 2.78[1.30-5.95] \\ \text { Lu } & 2009 & 1.5 & 1.94[0.73-5.15] \\ \text { Kuroda } & 2005 & 2.5 & 1.38[0.88-2.15] \\ \text { Mamot } & 2005 & 2.5 & 1.78[1.16-2.73] \\ \text { Mamot } & 2002 & 1 & 12.77[4.50-36.17] \\ \text { Prasad } & 2012 & 1.6 & 2.65[1.67-4.21] \\ \text { Pechman } & 2012 & 1.6 & 1.71[0.42-7.04] \\ \text { Pechman } & 2012 & 1.6 & 2.59[0.55-12.23] \\ \text { Pechman } & 2016 & 6.7 & 3.77[0.41-34.43] \\ \text { Ramachandran } & 2010 & 10 & 1.32[1.28-1.36] \\ \text { Global } & & & \\ \quad & & \mathbf{1 6} & \mathbf{3 . 2 1}[\mathbf{1 . 9 9 - 5 . 1 8}]\end{array}$

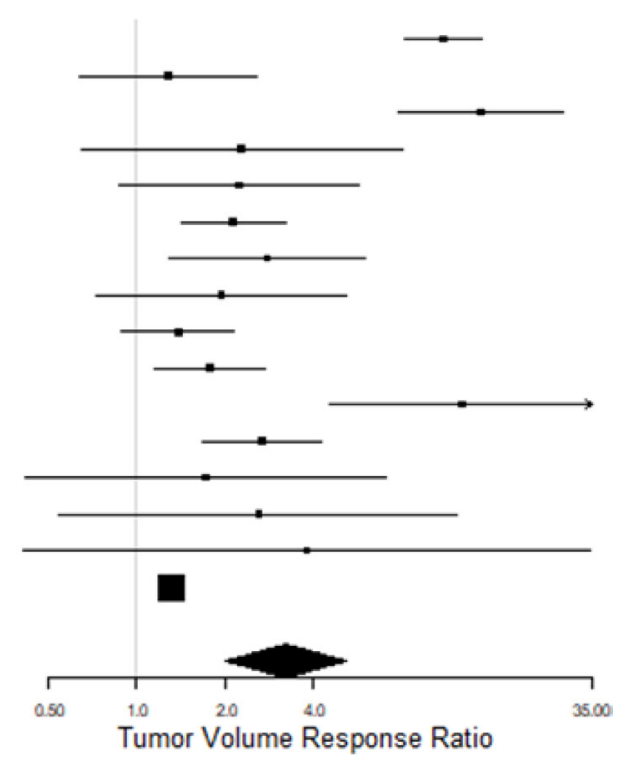

Figure 2: Meta-analysis of included studies based on outcome measure. (A) Meta-analysis of studies assessing animal survival. (B) Meta-analysis of experimental comparisons assessing tumor volume reduction. The grey line represents neutral effect. The $\mathrm{x}-\mathrm{axis}$ is shown in log scale. 
Table 2: Multivariable meta-regression

\begin{tabular}{lcc}
\hline Variables & $\boldsymbol{F}$ & $\boldsymbol{p}$ \\
\hline Model & 6.08 & $0.0005^{*}$ \\
Type of control & 3.85 & $0.0185^{*}$ \\
Route of drug delivery & 8.61 & $0.001^{*}$ \\
Drug & 3.74 & $0.0207^{*}$ \\
Quality score & 0.91 & 0.3482 \\
\hline
\end{tabular}

Multivariable meta-regression of predictors identified a priori.

Legend: ${ }^{*} p$-value $<0.05$.

$=0.7, p>0.0042$ ) was not significantly associated to an improved survival outcome in the univariable analysis. Drug dose for epirubicin and topotecan were not assessed due to low number included in the meta-analysis. The type of control, included into the multivariable analysis because of a large $F$-value on univariable analysis $(F=9.94, p<$ 0.0042 ), was also predictive of outcome on multivariable meta-regression $(F=3.85, p<0.05)$. Studies where the control was untreated or given a carrier were associated with greater efficacy than those controlled with vehicle or saline (Figure 3C).

All 6 glioma models used in survival experiments were associated with treatment efficacy (Figure 3D). The choice of glioma model was associated with outcome heterogeneity on multivariable meta-regression $(F=$ $6.08, p<0.05)$, with human GBM (1.58, 1.22-2.07) and rat $101 / 8(1.63,1.34-1.97)$ cell lines associated with greater efficacy than the most frequently reported U87 cell line $(1.14,0.90-1.43)$. Tumor model selection

A

$\begin{array}{lrr} & \mathbf{N} & \mathbf{E S}[\mathbf{9 5} \% \mathbf{C l}] \\ \text { Doxorubicin } & 28 & 1.29[1.17-1.43] \\ \text { Epirubicin } & 2 & 1.78[1.19-2.68] \\ \text { Irinotecan } & 14 & 1.38[1.16-1.65] \\ \text { Topotecan } & 3 & 1.17[0.85-1.61] \\ \text { Global } & \mathbf{4 7} & \mathbf{1 . 3 2}[\mathbf{1 . 2 3}-\mathbf{1 . 4 3}]\end{array}$

B $\begin{array}{lrr} & \text { N } & \text { ES }[95 \% \text { Cl] } \\ \text { Intraperitoneal } & 6 & 1.17[0.96-1.44] \\ \text { Intravenous } & 29 & 1.26[1.01-1.58] \\ \text { Intracranial } & 12 & 1.57[1.22-2.01] \\ \text { Global } & \mathbf{4 7} & \mathbf{1 . 3 2}[\mathbf{1 . 2 3}-\mathbf{1 . 4 3}]\end{array}$

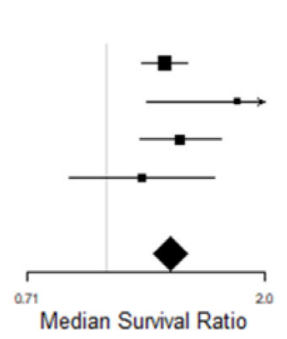

D

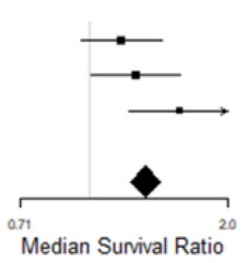

C was not associated with heterogeneity on univariable meta-regression $(F=3.08, p>0.0042)$. There were no associations between total study quality score and survival outcome on either multivariable $(F=0.91, p>0.05)$ or univariable $(F=0.18, p>0.0042)$ analysis.

The remaining variables were tested on univariable meta-regression only (Table 3, Figure 4). There were no associations between survival outcome and frequency of drug administration $(F=1.68, p>0.0042)$, site of tumor implantation $(F=2.32, p>0.0042)$, species $(F=1.68, p$ $>0.0042)$, comorbidity $(1.14, p>0.0042)$, the reporting of randomised group allocation $(F=0.79, p>0.0042)$ or blinded assessment of outcome $(F=0.79, p>0.0042)$.

\section{Tumor volume dataset}

As there were only 16 experiments included in this meta-analysis, we did not apply multivariate metaregression. All 16 experiments used a U87 glioma model

$\begin{array}{lrr}\text { Carrier } & 10 & 1.41[1.24-1.59] \\ \text { Saline } & 17 & 1.12[0.95-1.32] \\ \text { Vehicle } & 5 & 1.07[0.86-1.34] \\ \text { Untreated } & 15 & 1.64[1.39-1.94]\end{array}$

Global

\section{$47 \quad 1.32[1.23-1.43]$}

D

$\begin{array}{lrr}\text { 101/8 } & 6 & 1.63[1.34-1.97] \\ \text { 9L } & 8 & 1.40[1.08-1.80] \\ \text { BT4Ca } & 5 & 1.20[0.91-1.59] \\ \text { GBM } & 7 & 1.59[1.22-2.07] \\ \text { U251 } & 7 & 1.24[0.94-1.63] \\ \text { U87 } & 14 & 1.14[0.90-1.43] \\ & & \\ \text { Global } & \mathbf{4 7} & \mathbf{1 . 3 2}[\mathbf{1 . 2 3}-\mathbf{1 . 4 3}]\end{array}$

Global $\quad 47 \quad 1.32[1.23-1.43]$
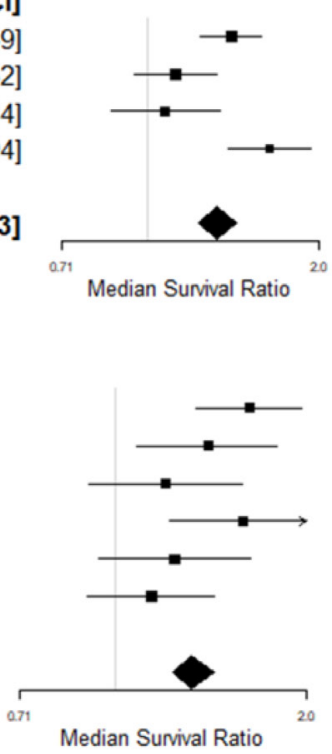

Figure 3: Multivariable meta-regression based on survival outcome. Forest plots of (A) choice of drug, (B) route of drug delivery, (C) type of control and, (D) choice of glioma model for the study. Median survival ratio was calculated by dividing the mean outcome of the treatment groups by the mean outcome of the control group. The solid grey line represents neutral effect. The $\mathrm{x}$-axis is shown in log-scale. 
Table 3: Univariable meta-regression

\begin{tabular}{|c|c|c|c|c|}
\hline \multirow[b]{2}{*}{ Variables } & \multicolumn{2}{|c|}{ Survival } & \multicolumn{2}{|c|}{ Tumor volume } \\
\hline & $F$ & $p$ & $F$ & $p$ \\
\hline Animal species & 1.68 & 0.2011 & - & - \\
\hline Model & 3.05 & 0.0198 & na & na \\
\hline Comorbidity & 1.14 & 0.3526 & - & - \\
\hline Site of tumor implantation & 2.32 & 0.1349 & 2.47 & 0.1386 \\
\hline Drug & 1.14 & 0.3448 & 1.05 & 0.405 \\
\hline Route of drug delivery & 3.92 & 0.0271 & 1.17 & 0.3601 \\
\hline Type of control & 9.94 & $<0.0001^{*}$ & 0.29 & 0.8779 \\
\hline Frequency of administration & 1.07 & 0.3518 & 0.21 & 0.8168 \\
\hline Randomisation & 0.79 & 0.3801 & 0.06 & 0.8146 \\
\hline Blinded outcome assessment & 0.79 & 0.3785 & na & na \\
\hline Quality score & 0.18 & 0.671 & 0.81 & 0.691 \\
\hline
\end{tabular}

Comparison of univariable meta-regression for survival and tumor volume experimental comparisons.

Legend: ${ }^{*} p$-value < 0.05; na - not applicable.

reported and therefore we could not apply glioma model to a univariable meta-regression. Similarly, the majority of studies used mice $(13 / 16)$ and athymic animals (14/16), and all were not blinded. Consequently, we did not test species, comorbidity or blinded assessment of outcome. None of the remaining variables, when applied to univariable meta-regression (Table 3, Figure 5 ), were found to be predictive of volume outcome,

A $\begin{array}{lrr} & \text { N } & \text { ES [95\% Cl] } \\ \text { Continuous } & 1 & 0.95[0.57-1.57] \\ \text { Single } & 36 & 1.32[0.79-2.20] \\ \text { Multiple } & 10 & 1.40[0.82-2.37] \\ \text { Global } & \mathbf{4 7} & \mathbf{1 . 3 2}[\mathbf{1 . 2 3}-\mathbf{1 . 4 3}]\end{array}$

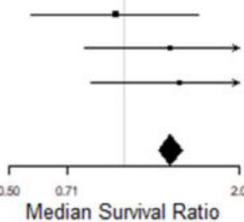

B $\begin{array}{lrr}\text { B } & \text { N } & \text { ES [95\% Cl] } \\ \text { Intracranial } & 44 & 1.35[1.24-1.46] \\ \text { Subcutaneous } & 3 & 1.06[0.77-1.44] \\ \text { Global } & \mathbf{4 7} & \mathbf{1 . 3 2}[\mathbf{1 . 2 3}-\mathbf{1 . 4 3}]\end{array}$

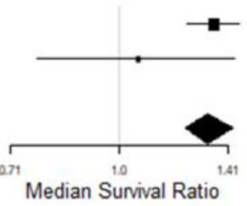

C

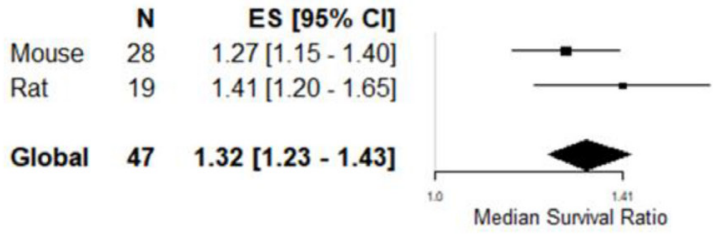

including drug $(F=1.05, p>0.0056)$, doxorubicin dose $(F=0.24, p>0.0056)$, irinotecan dose $(F=0.04, p>$ $0.0056)$, route of delivery $(F=1.17, p>0.0056)$, type of control $(F=0.29, p>0.0056)$, frequency of drug administration $(F=0.21, p>0.0056)$, site of tumor implantation $(F=2.47, p>0.0056)$, randomised group allocation $(F=0.06, p>0.0056)$ and quality score $(F=$ $0.81, p>0.0056)$.

$\begin{array}{lrr}\text { D } & \text { N } & \text { ES }[95 \% \text { Cl] } \\ \text { Athymic } & 24 & 1.25[1.12-1.39] \\ \text { RAG2-M } & 2 & 1.18[0.78-1.79] \\ \text { SCID } & 2 & 1.68[1.11-2.53] \\ \text { None } & 1 & 1.39[1.18-1.64] \\ \text { Unknown } & 18 & 1.80[1.02-3.18] \\ & & \\ \text { Global } & \mathbf{4 7} & \mathbf{1 . 3 2}[\mathbf{1 . 2 3}-\mathbf{1 . 4 3}]\end{array}$

$\mathbf{E}$

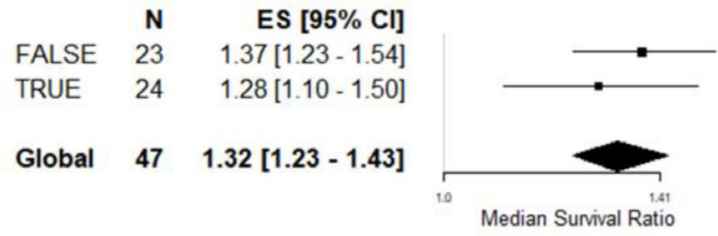

F

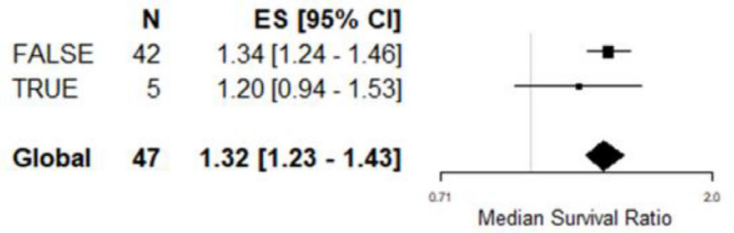

Figure 4: Univariable meta-regression based on survival outcome. Forest plots of (A) frequency of drug administration, (B) tumor site implantation, (C) animal species, (D) comorbidity, (E) randomisation, and (F) blinding of outcome assessment. The solid grey line represents neutral effect. The $\mathrm{x}$-axis is shown in log-scale. 


\section{Post-hoc assessment of drug carriers}

Two of the significant results from the two analyses pertained to drug delivery - by way of route of delivery directly and indirectly in the way of the type of control used. We have observed variance in the use of drug carriers already and it is possible that the use and choice of carriers could affect both of these significant results. As such, we thought it important to describe the frequency of carrier use (Supplementary Table 3), and have subsequently included this stratification post-hoc in the univariable metaregression.

Of the 52 publications included in the systematic review, $58 \%(n=30)$ used a carrier for drug delivery. The most frequently reported carriers were nanoparticles ( $n$ $=8)$ and liposomes $(n=7)$ as drug carriers all of which were administer intravenously except for one study using nanoparticles that administered via intraperitoneal route. Other carriers observed were polymers $(n=6)$, drug eluting beads $(n=3)$, nanoliposomes $(n=2)$, albumin $(n=1)$, micelles $(n=1)$, microbubbles $(n=1)$, and microspheres $(n=1)$. Of the 38 publications included in the meta-analysis, 25 used a carrier intended to enhance drug delivery into the brain. The choice of carrier was not associated with heterogeneity in the survival $(F=2.04$, $p=0.0824)$ or volume $(F=0.95, p=0.472)$ datasets on univariable meta-regression (Figure 6).

\section{Publication bias}

Taking all studies included in the systematic review, we found evidence of publication bias in both datasets. For the survival dataset, publication bias was observed by means of an asymmetric funnel plot (Figure 7A), and a significantly positive intercept in the Egger's regression test ( $B=11.24, t=8.67, p<0.001$, Figure 7B). We observed dramatic funnel plot asymmetry in the volume dataset (Figure 7C), although Egger's regression test did not show a significantly positive intercept $(B=0.377$, $t=0.151, p>0.05$, Figure 7D). Trim and Fill analysis suggested the presence of 14 'missing' studies in the tumor volume dataset (Figure 7C): addition of the 'missing' studies dramatically reduced overall efficacy from 2.35 to 1.35 . We applied a contour overlay to the funnel plot (Figure 7C) to help identify the cause of funnel plot asymmetry [37]. In this case, the imputed 'missing' tumor
A

$\begin{array}{lrr} & \text { N } & \text { ES [95\% Cl] } \\ \text { Doxorubicin } & 5 & 2.94[1.32-6.56] \\ \text { Epirubicin } & 1 & 1.78[0.26-12.11] \\ \text { Etoposide } & 1 & 14.21[1.99-101.37] \\ \text { lrinotecan } & 9 & 3.03[1.06-8.60] \\ \text { Global } & \mathbf{1 6} & \mathbf{3 . 2 1}[\mathbf{1 . 9 9 - 5 . 1 8}]\end{array}$

B

Intraperitoneal
Intravenous
Oral
Subcutaneous
Global

C

$\begin{array}{lrr} & \text { N } & \text { ES [95\% Cl] } \\ \text { Carrier } & 1 & 2.12[0.31-14.78] \\ \text { Saline } & 6 & 3.45[0.42-28.37] \\ \text { Vehicle } & 2 & 2.84[0.41-19.79] \\ \text { Untreated } & 4 & 2.38[0.24-23.73] \\ \text { Unknown } & 3 & 5.94[0.53-66.88] \\ & & \\ \text { Global } & 16 & \mathbf{3 . 2 1}[1.99-5.18]\end{array}$

ES [ $[95 \% \mathrm{Cl}]$

$3.63[1.62-8.16]$

$2.66[0.91-7.76]$

$14.21[2.01-100.47]$

$2.04[0.45-9.34]$

$3.21[1.99-5.18]$

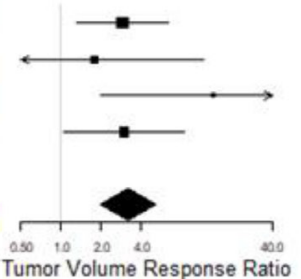

D

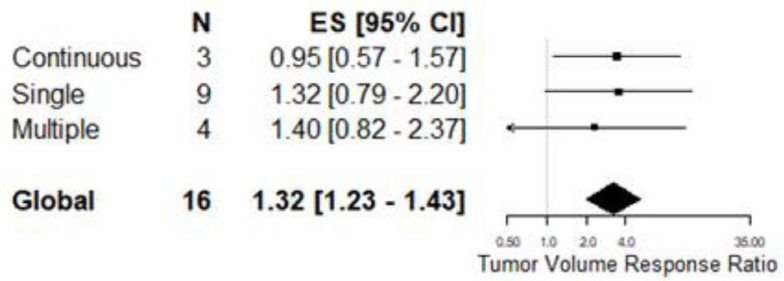

$\mathbf{E}$

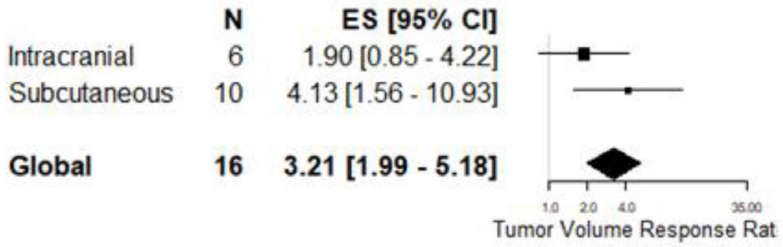

F

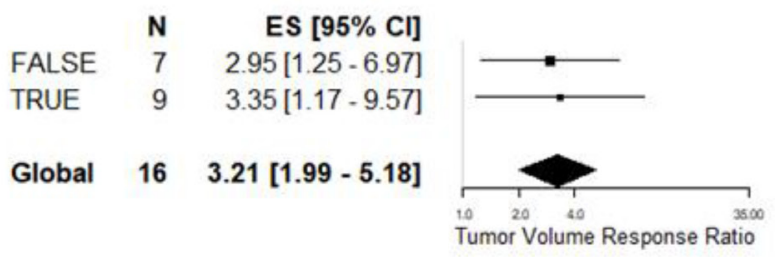

Figure 5: Univariable meta-regression based on tumour volume reduction. Forest plots of (A) drug of choice, (B) route of administration, (C) type of carrier, (D) frequency of administration, (E) site of tumor implantation, and (F) randomisation. The solid grey line represents neutral effect. The $\mathrm{x}$-axis is shown in log-scale. 
volume studies are equally distributed between the areas of high and non-statistical significance.

\section{DISCUSSION}

In this study, we report the efficacy of five different drugs - doxorubicin, epirubicin, etoposide, irinotecan and topotecan - in pre-clinical glioma models. Fifty-two publications (90 comparisons) satisfied our inclusion criteria. Of these, 38 publications with 63 experimental comparisons consisting of 1,192 animals for treatment efficacy was used for the meta-analysis. Study design was heterogeneous and the risk of bias was high.

Nonetheless, we observed that efficacy estimates favoured treatment in all drugs in both survival and tumor volume data. We have applied a multivariable meta-regression which suggests that glioma model, drug choice, route of drug delivery and type of control are each independently associated with survival outcome in this preclinical setting.

\section{Validity of statistical approach}

In previous reviews of the glioma literature [1416], a large number of significant results on stratified meta-analysis and a particularly large range of efficacies observed across a number of glioma models has generated suspicion of collinearity and, as such, rendered results difficult to interpret.

We therefore devised two strategies to counter this: firstly, to exclude infrequently reported glioma models (those reported in fewer than five experiments), and secondly to apply multivariable meta-regression to the remaining data following a standard (univariable) metaregression. The univariable meta-regression returned fewer significant findings than previous stratified metaanalyses in glioma studies, probably because metaregression is more conservative than stratified metaanalysis [38, 39]; and because we included fewer studies. Based on the multivariable meta-regression analysis, four variables were independently associated with survival heterogeneity. Three of these, namely glioma model,

A
Albumin
Drug Eluting Beads
Liposome
Micelles
Nanoparticles
Polymer
NA
Global
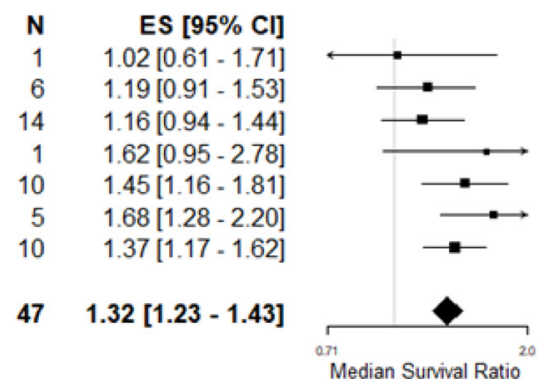

B

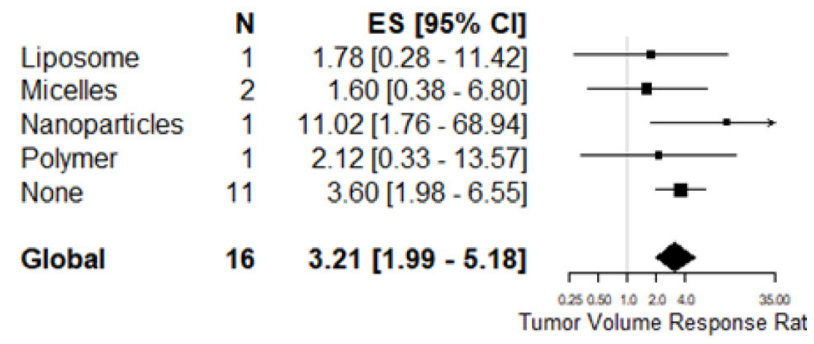

drug and route of drug delivery were nonsignificant on univariable meta-regression. The revelation of these associations on multivariable modelling confirms the presence of collinearity in this data as suspected, and thus validates multivariable modelling in this context.

We suggested that the selection of carrier may have had confounded analyses of route of delivery and control used, both of which returned significant results meta-regression, and on this basis, we felt it appropriate to include carrier in univariable meta-regression post hoc. While this result was non-significant, we did not include in a multivariable model and so it remains possible that a confounding effect of drug carriers may exist. We observed no significant associations on the univariable analysis of the tumor volume dataset, and this is likely a manifestation of a large degree of heterogeneity, collinearity and small sample size.

\section{Impact of factors relating to study quality}

On further inspection of the included studies we found a large range of experimental design, relating to both design parameters and measures to reduce the risk of bias. Randomisation and blinded assessment of outcome are important measures of overall study quality and inflated treatment efficacy in the context of a lack thereof is a phenomenon well-described in the pre-clinical neurosciences literature [40] and in previous glioma studies $[15,16]$. Infrequent reporting of randomization and blinding in this dataset is consistent with other studies although we have not observed an association with experimental outcome. This may be because of collinearity or small sample size, and might be revealed in a larger multivariable meta-regression of glioma studies.

Furthermore, we have found evidence of publication bias in both datasets by way of asymmetrical Funnel plots and a positive Egger's regression intercept in the survival dataset and 'missing studies' found in Trim and Fill analysis of the tumor volume dataset. These suggest that there is a relative lack of small, inefficacious studies that are not reported for several reasons [41] and resulting in a consequent inflation of efficacy perceived both in subjective impressions of the literature and in this meta-

Figure 6: Post-hoc univariable meta-regression analysis of choice of carriers. Post-hoc univariable analysis of carriers used in studies assessing (A) survival and (B) tumor volume reduction. 
analysis. Contour enhancement of the funnel plot suggests that the asymmetry observed in the tumor volume dataset may also be due to other confounding factors rather than publication bias alone.

\section{Factors relating to study design}

Animal experiments may often be viewed as a stepping stone from bench to bedside, given the innate similarities between both hosts and their diseases in physiological and genetic terms. Unfortunately, animal models have several key features that, although not preclusive of their value in research, render them imperfect.

Animal experiments are often designed to minimize variables and thus tend to report the use of genetically similar individuals seeded with tumors derived from a single cell line. Immortalized cell lines used to produce

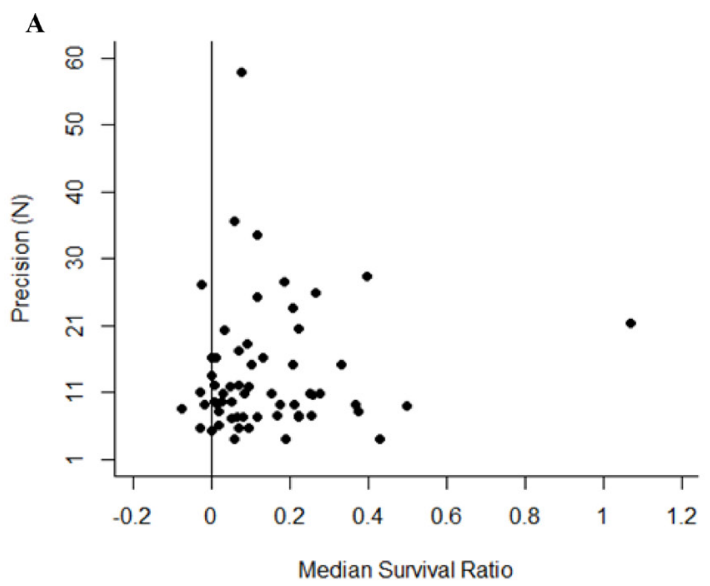

B

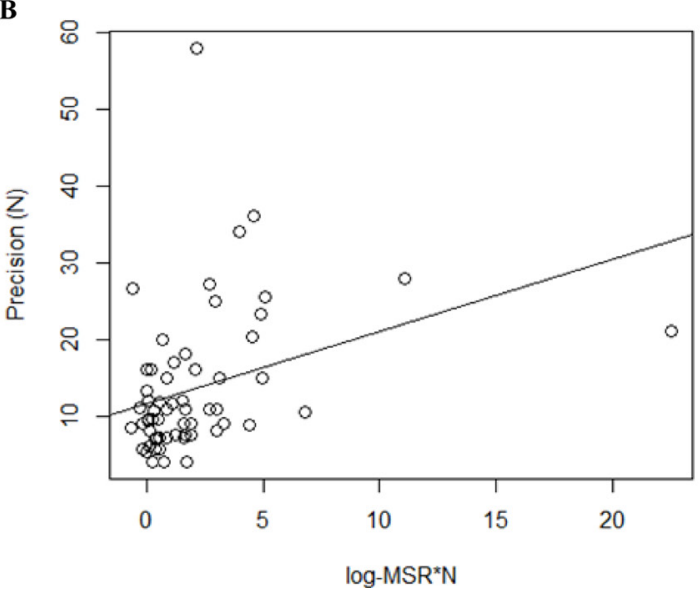

xenograft models are well established and easy to cultivate, although have several key limitations. An example is the most commonly used glioma xenograft - U87 - which has been observed to have a distinct DNA profile and grow in a pattern not typical of a human GBM [42-44]. They are genetically homogenous and behave predictably - thus lacking heterogeneity observed in GBM patients. Pooling of studies in meta-analysis provides a way to simulate this heterogeneity and retest a treatment in this context. However, its value is dependent on the underpinning data - in terms of quality and the experiment's ability to replicate the biology and heterogeneity of human disease. An example of note in this context is the TP53 status of tumour model used in the studies in this analysis. Of the six GBM models found in 70 experimental comparisons included in the meta-analysis, four have known TP53 mutation status but only one study screened for this. Based on trend, better response can be observed on TP53
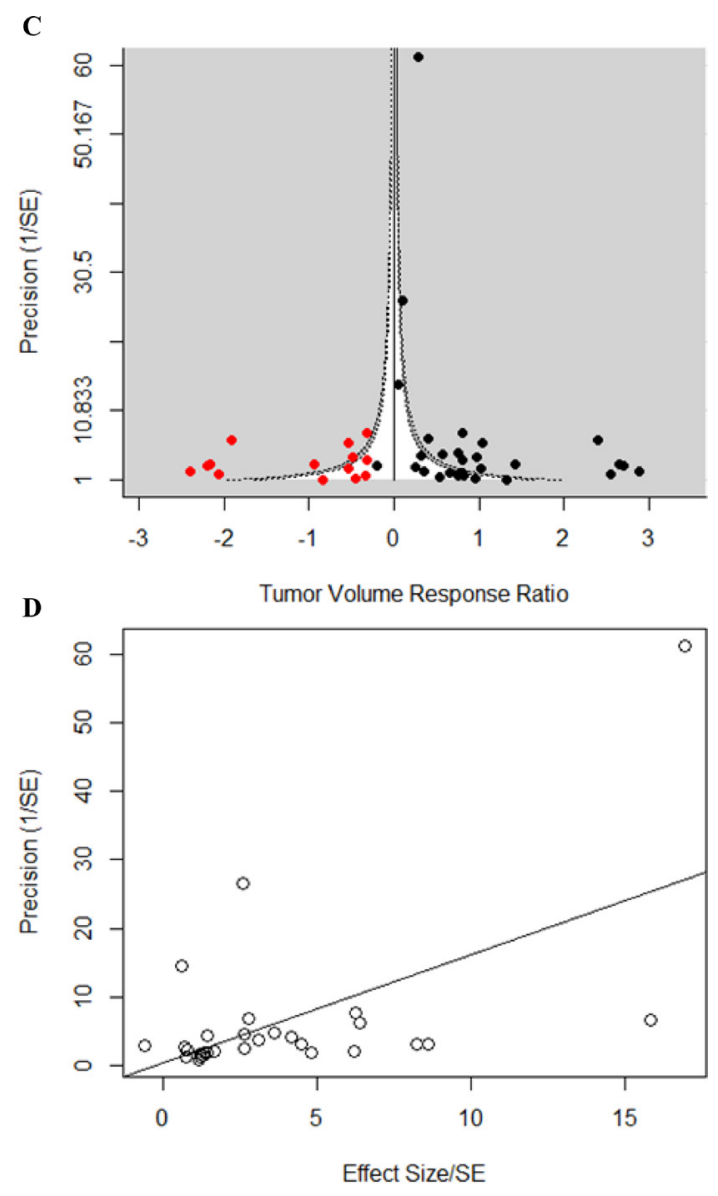

Figure 7: Publication bias. Funnel plots and Egger's regression publication bias plot for all studies included in the systematic review for both (A and B) survival and (C and D) tumor volume datasets. Survival studies show significant publication bias $(p<0.001)$ as shown by the asymmetry in (A) and by means of a positive intercept (11.24) in (B). For the tumor volume studies, publication bias was observed by means of funnel plot asymmetry observed in (C). Trim and fill imputed 14 'missing' studies (red dots) thus reducing overall efficacy estimate in (C). Egger's regression analysis of the tumor volume dataset did not return a significantly positive intercept (D). Funnel plots show the effect size (x-axis) against study precision (y-axis). Egger's regression publication bias plot shows the standardised effects size (x-axis) against study precision (y-axis). Solid black lines in (A) and (B) represent the level of neutral effect. Funnel plot in (C) is contourenhanced. The light grey shading represents area of high statistical significance, while the white area represents the area of non-statistical significance. The darker shaded contour line represents the area of associated statistical significance $(p=0.05)$. 
wildtype cell lines (Table 1, Figure 3D). We have shown that glioma model selection is one of the most important predictors of treatment efficacy; more so than features such as drug of choice and several quality parameters.

A discrepancy we have observed between this metaanalysis and a similar review of human clinical trials is the absence of doxorubicin [34], contrasting with animal studies in which it was the most commonly used drug. Glioma model selection should be considered very carefully in the design phase of future studies to improve translation of pre-clinical investigations to human clinical use.

In this study we have not pursued the effects of the large range of dosing schedules used. Our primary intention was to review the overall efficacy of topoisomerase inhibitors rather than to tease out the intricacies of dose scheduling. Furthermore, to do this would require a larger dataset to permit analysis of timing and duration of treatment, dosage, frequency and number of cycles with sufficient power. To provide a rough insight we used total dose, which was not associated with heterogeneity for doxorubicin or irinotecan in either dataset.

\section{Limitations of this review}

We believe the conclusions drawn from this study are important, however these should be taken with caution due to a number of limitations. Although meta-analysis is a powerful tool, it comes with its weaknesses. It is at the mercy of the rigour in which it is undertaken, and we have minimised this through transparent reporting and prior publication of a protocol. Nonetheless, our search terms only included generic drug names (e.g. irinotecan). In omitting older synonyms, such as CPT-11, we may have missed a small number of older studies. The findings of a meta-analysis are only as reliable as the primary data included - we found that quality was overall low, which should be acknowledged when interpreting the results of this study. Meta-analysis is a tool to observe associations between study design parameters and efficacy; these findings do not imply causality, so should only be considered as hypothesis-generating. While we have identified and attempted correction for a number of confounding factors, further unacknowledged confounders could still have distorted our results. An example of this is the carrier used for drug delivery, which we did not prespecify as a variable of interest in our protocol. When a confounding effect subsequently became mechanistically and statistically plausible, we were reluctant to include in the multivariable model in the interest of preserving the integrity of our analysis. Findings and questions arising from this review should be answered with prospective, high-quality studies.

\section{CONCLUSIONS}

Topoisomerase inhibition has failed to prove successful in clinical trials despite apparently consistent efficacy in preclinical studies. However, a number of concerns arise relating to the internal and construct validity of the animal literature: the existing literature is at high risk of bias, with evidence of augmented perceived treatment efficacy, and animal research has limitations in the recapitulation of human disease. Nonetheless, factors such as glioma model, type of control used, route of administration and type of drug used appear to predict outcome and must be taken into consideration when planning future studies. We believe that further highquality, prospective in vivo studies accommodating these conclusions would be invaluable in helping to further define the role for topoisomerase inhibition in clinical GBM.

\section{MATERIALS AND METHODS}

\section{Experimental design}

We aimed to undertake a systematic review and meta-analysis of topoisomerase inhibition in preclinical glioma models; outcome measures of interest were changes in survival and tumour volume. Furthermore, we anticipated significant heterogeneity between studies, which we investigated using meta-regression, and publication bias, which we have investigated using funnel plots, Egger's regression test and Trim and Fill analysis.

This was conducted as described in a protocol, published online on 15 October 2015. Available at http:// www.den.ed.ac.uk/camarades/research.html\#protocols.

\section{Sources}

A literature search of phase II clinical trials revealed that doxorubicin, epirubicin, etoposide, irinotecan and topotecan are the most commonly used FDA-approved topoisomerase inhibitors for the treatment of GBM. In August 2014, we searched PubMed, Medline and Embase for the following keywords: (glioblastoma or glioblastoma multiforme or GBM or high-grade glioma) AND (Doxorubicin OR Epirubicin OR Etoposide OR Irinotecan OR Topotecan). The search was limited to in vivo studies with predeveloped filters $[45,46]$, with no language or publication date restrictions. The search was updated in July 2016.

\section{Inclusion criteria}

During the screening process, criteria for inclusion required the following information from the studies: (i) a topoisomerase inhibitor used as monotherapy, (ii) use of an adult high-grade glioma model, (iii) intracranial or subcutaneous tumor implantation and (iv) tumor volume or median survival reported as the outcome measure. The title and abstracts were screened by two of the authors (TJ and $\mathrm{TH}$ ) for inclusion. 


\section{Data extraction}

All data extracted were entered into the CAMARADES data manager application. Data were extracted regarding the publication (author names, year of publication, and title), intervention (drug used, dose, dose frequency, route of administration, drug delivery method, delay to treatment, and time of assessment based on day 0 of treatment), animal population (species, strain, sex, age, and number of animals per group), tumor implantation (cell type used, site of implantation, number or volume of cells inoculated and inoculation method used) and outcome measures (tumor volume and median survival data). All data used in this study is available in Supplementary Dataset 1.

The median survival and tumor volume data were estimated from graphs using a desktop ruler when these were not provided in the text.

\section{Study quality scoring}

A modified 12-item checklist adapted from previously published studies was used to assess the quality of the studies and determine possible publication bias (Supplementary Table 2) [14-16].

\section{Statistical analysis}

A summary statistic was initially performed for each individual study. For survival data, we used median survival ratio as described previously [47] and for tumor volume data we used response ratio [48]. Data were then normalized via $\log$ transformation and pooled using DerSimonian and Laird random-effects meta-analysis. We weighted survival studies by the number of animals in the study as a surrogate marker for inverse variance [47].

We assessed for the presence of heterogeneity using the $I^{2}$ statistic and investigated sources of heterogeneity using meta-regression (via the metareg command in STATA 12.0). To limit the covariance observed in glioma models, we excluded those studies using glioma models reported in fewer than five experiments. We then performed a univariable meta-regression followed by a multivariable meta-regression where sufficient data were present; further details are described in the results section. Bonferroni correction was applied on the comparisons in the univariable meta-regression; critical $p$-value was adjusted to $p=0.0042$ for survival data (12 comparisons) and $p=0.0056$ for volume data (nine comparisons). In multivariable meta-regression, critical $p$-value was set at 0.05 . Publication bias was assessed using contourenhanced funnel plots, Egger's regression test and 'Trim and Fill' analysis using 'foresplot' and 'metafor' $\mathrm{R}$ packages [37, 49-51]. Contour enhancement of funnel plots was performed to help identify cause of funnel plot asymmetry [37]. All tests reported are two-sided.

\section{Author contributions}

conception (TJ, KM), design (TJ, ES, MM, TH), literature search (TJ), study screening (TJ, TH), data extraction (TJ), data analysis (TH, TJ), interpretation (TJ, ES, MM, KM, TH), write up (TJ, TH, MM, KM).

\section{ACKNOWLEDGMENTS}

We would like to acknowledge Ms. Martina Hochwartner for assisting with the translation of some of the articles. We thank, Peter-Paul Zwetsloot of the University Medical Centre Utrecht for assistance with multivariable analysis techniques. Translational Cancer Research Network (TCRN) at UNSW Sydney for assistance with data extraction and the travel grant to present the initial results to the 5th quadrennial meeting of the World Federation of Neuro-Oncology Societies (WFNOS).

\section{CONFLICTS OF INTEREST}

The authors have no potential conflicts of interest.

\section{FUNDING}

This work was supported by the UK National Centre for the Replacement, Refinement and Reduction of Animals in Research (NC3Rs) infrastructure award: ivSyRMAF - the CAMARADES - NC3Rs in vivo systematic review and meta-analysis facility $(\mathrm{NC} /$ L000970/1), Prince of Wales Clinical School (UNSW Australia) and Cure Brain Cancer Foundation. The funders had no role in the study design, collection and analysis of data, decision of publish or preparation of the manuscript.

\section{REFERENCES}

1. Ostrom QT, Gittleman H, Xu J, Kromer C, Wolinsky Y, Kruchko C, Barnholtz-Sloan JS. CBTRUS Statistical Report: Primary Brain and Other Central Nervous System Tumors Diagnosed in the United States in 2009-2013. Neuro-Oncology. 2016; 18:v1-v75. https://doi.org/10.1093/ neuonc/now207.

2. Paszat L, Laperriere N, Groome P, Schulze K, Mackillop W, Holowaty E. A population-based study of glioblastoma multiforme. Int J Radiat Oncol Biol Phys. 2001; 51:100-7. https://doi.org/10.1016/S0360-3016(01)01572-3.

3. Chakrabarti I, Cockburn M, Cozen W, Wang YP, PrestonMartin S. A population-based description of glioblastoma multiforme in Los Angeles County, 1974-1999. Cancer. 2005; 104:2798-806. https://doi.org/10.1002/cncr.21539.

4. Ostrom QT, Gittleman H, de Blank PM, Finlay JL, Gurney JG, McKean-Cowdin R, Stearns DS, Wolff JE, Liu M, 
Wolinsky Y, Kruchko C, Barnholtz-Sloan JS. American Brain Tumor Association Adolescent and Young Adult Primary Brain and Central Nervous System Tumors Diagnosed in the United States in 2008-2012. Neuro Oncol. 2016; 18:11-i50. https://doi.org/10.1093/neuonc/nov297.

5. Jue TR, Hovey E, Davis S, Carleton O, McDonald KL. Incorporation of biomarkers in phase II studies of recurrent glioblastoma. Tumour Biol. 2015; 36:153-62. https://doi. org/10.1007/s13277-014-2960-3.

6. Stupp R, Mason WP, van den Bent MJ, Weller M, Fisher B, Taphoorn MJ, Belanger K, Brandes AA, Marosi C, Bogdahn U, Curschmann J, Janzer RC, Ludwin SK, et al. Radiotherapy plus Concomitant and Adjuvant Temozolomide for Glioblastoma. New England Journal of Medicine. 2005; 352:987-96. https://doi.org/10.1056/ NEJMoa043330.

7. McDonald KL, Rapkins RW, Olivier J, Zhao L, Nozue K, Lu D, Tiwari S, Kuroiwa-Trzmielina J, Brewer J, Wheeler HR, Hitchins MP. The T genotype of the MGMT C $>$ T (rs16906252) enhancer single-nucleotide polymorphism (SNP) is associated with promoter methylation and longer survival in glioblastoma patients. Eur J Cancer. 2013; 49:360-8. https://doi.org/10.1016/j.ejca.2012.08.012.

8. Rapkins RW, Wang F, Nguyen HN, Cloughesy TF, Lai A, Ha W, Nowak AK, Hitchins MP, McDonald KL. The MGMT promoter SNP rs16906252 is a risk factor for MGMT methylation in glioblastoma and is predictive of response to temozolomide. Neuro Oncol. 2015; 17:1589-98. https://doi. org/10.1093/neuonc/nov064.

9. Hegi ME, Diserens AC, Gorlia T, Hamou MF, de Tribolet N, Weller M, Kros JM, Hainfellner JA, Mason W, Mariani L, Bromberg JE, Hau P, Mirimanoff RO, et al. MGMT Gene Silencing and Benefit from Temozolomide in Glioblastoma. New England Journal of Medicine. 2005; 352:997-1003. https://doi.org/10.1056/NEJMoa043331.

10. Jue TR, McDonald KL. The challenges associated with molecular targeted therapies for glioblastoma. Journal of Neuro-Oncology. 2016; 127:427-34. https://doi. org/10.1007/s11060-016-2080-6.

11. O'Rourke DM, Nasrallah MP, Desai A, Melenhorst JJ, Mansfield K, Morrissette JJD, Martinez-Lage M, Brem S, Maloney E, Shen A, Isaacs R, Mohan S, Plesa G, et al. A single dose of peripherally infused EGFRvIII-directed CAR $T$ cells mediates antigen loss and induces adaptive resistance in patients with recurrent glioblastoma. Science Translational Medicine. 2017; 9. https://doi.org/10.1126/ scitranslmed.aaa0984.

12. Macleod MR, McLean AL, Kyriakopoulou A, Serghiou S, de Wilde A, Sherratt N, Hirst T, Hemblade R, Bahor $\mathrm{Z}$, Nunes-Fonseca $\mathrm{C}$. Risk of bias in reports of in vivo research: a focus for improvement. PLoS Biol. 2015; 13: e1002273. https://doi.org/10.1371/journal.pbio.1002273.

13. Sena ES, Briscoe CL, Howells DW, Donnan GA, Sandercock PAG, Macleod MR. Factors Affecting the Apparent Efficacy and Safety of Tissue Plasminogen
Activator in Thrombotic Occlusion Models of Stroke: Systematic Review and Meta-Analysis. Journal of Cerebral Blood Flow \& Metabolism. 2010; 30:1905-13. https://doi. org/10.1038/jcbfm.2010.116.

14. Amarasingh S, Macleod MR, Whittle IR. What is the translational efficacy of chemotherapeutic drug research in neuro-oncology? A systematic review and meta-analysis of the efficacy of BCNU and CCNU in animal models of glioma. J Neurooncol. 2009; 91:117-25. https://doi. org/10.1007/s11060-008-9697-z.

15. Hirst TC, Vesterinen HM, Sena ES, Egan KJ, Macleod MR, Whittle IR. Systematic review and meta-analysis of temozolomide in animal models of glioma: was clinical efficacy predicted? Br J Cancer. 2013; 108:64-71. https:// doi.org/10.1038/bjc.2012.504.

16. Hirst T, Vesterinen H, Conlin S, Egan K, Antonic A, Lawson McLean A, Macleod M, Grant R, Brennan P, Sena E. A systematic review and meta-analysis of gene therapy in animal models of cerebral glioma: why did promise not translate to human therapy? Evidence-based Preclinical Medicine. 2014; 1:21-33. https://doi.org/10.1002/ebm2.6.

17. Søe K, Grosse F. p53 stimulates human topoisomerase I activity by modulating its DNA binding. Nucleic Acids Research. 2003; 31:6585-92. https://doi.org/10.1093/nar/ gkg846.

18. Gobert C, Skladanowski A, Larsen AK. The interaction between p53 and DNA topoisomerase I is regulated differently in cells with wild-type and mutant p53. Proceedings of the National Academy of Sciences. 1999; 96:10355-60. https://doi.org/10.1073/pnas.96.18.10355.

19. Sandri MI, Isaacs RJ, Ongkeko WM, Harris AL, Hickson ID, Broggini M, Vikhanskaya F. p53 Regulates the Minimal Promoter of the Human Topoisomerase II $\alpha$ Gene. Nucleic Acids Research. 1996; 24:4464-70. https://doi.org/10.1093/ nar/24.22.4464.

20. Wang Q, Zambetti GP, Suttle DP. Inhibition of DNA topoisomerase II alpha gene expression by the p53 tumor suppressor. Mol Cell Biol. 1997; 17:389-97. https://doi. org/10.1128/MCB.17.1.389.

21. Ataka M, Ikeguchi M, Yamamoto M, Inoue M, Tanida T, Oka S, Katano K. Topoisomerase I protein expression and prognosis of patients with colorectal cancer. Yonago Acta medica. 2007; 50:81-7.

22. Burden DA, Osheroff N. Mechanism of action of eukaryotic topoisomerase II and drugs targeted to the enzyme. Biochimica et Biophysica Acta. 1998; 1400:139-54. https:// doi.org/10.1016/S0167-4781(98)00132-8.

23. Cancer Genome Atlas Research Network. Comprehensive genomic characterization defines human glioblastoma genes and core pathways. Nature. 2008; 455:1061-8. https://doi. org/10.1038/nature07385.

24. Nagpal J, Jamoona A, Gulati ND, Mohan A, Braun A, Murali R, Jhanwar-Uniyal M. Revisiting the role of p53 in primary and secondary glioblastomas. Anticancer Res. 2006; 26:4633-9. 
25. Shiraishi S, Tada K, Nakamura H, Makino K, Kochi M, Saya H, Kuratsu J, Ushio Y. Influence of p53 mutations on prognosis of patients with glioblastoma. Cancer. 2002; 95:249-57. https://doi.org/10.1002/cncr.10677.

26. Reynes G, Martinez-Sales V, Vila V, Balana C, PerezSegura P, Vaz MA, Benavides M, Gallego O, Palomero I, Gil-Gil M, Fleitas T, Reche E. Phase II trial of irinotecan and metronomic temozolomide in patients with recurrent glioblastoma. Anticancer Drugs. 2016; 27:133-7. https:// doi.org/10.1097/cad.0000000000000314.

27. Whittle JR, Lickliter JD, Gan HK, Scott AM, Simes J, Solomon BJ, MacDiarmid JA, Brahmbhatt H, Rosenthal MA. First in human nanotechnology doxorubicin delivery system to target epidermal growth factor receptors in recurrent glioblastoma. J Clin Neurosci. 2015; 22:1889-94. https://doi.org/10.1016/j.jocn.2015.06.005.

28. Peters KB, Lou E, Desjardins A, Reardon DA, Lipp ES, Miller E, Herndon JE 2nd, McSherry F, Friedman HS, Vredenburgh JJ. Phase II Trial of Upfront Bevacizumab, Irinotecan, and Temozolomide for Unresectable Glioblastoma. Oncologist. 2015; 20:727-8. https://doi. org/10.1634/theoncologist.2015-0135.

29. Chauffert B, Feuvret L, Bonnetain F, Taillandier L, Frappaz D, Taillia H, Schott R, Honnorat J, Fabbro M, Tennevet I, Ghiringhelli F, Guillamo JS, Durando X, et al. Randomized phase II trial of irinotecan and bevacizumab as neo-adjuvant and adjuvant to temozolomide-based chemoradiation compared with temozolomide-chemoradiation for unresectable glioblastoma: final results of the TEMAVIR study from ANOCEFdagger. Ann Oncol. 2014; 25:1442-7. https://doi.org/10.1093/annonc/mdu148.

30. Hofland KF, Hansen S, Sorensen M, Engelholm S, Schultz HP, Muhic A, Grunnet K, Ask A, Costa JC, Kristiansen C, Thomsen C, Poulsen HS, Lassen U. Neoadjuvant bevacizumab and irinotecan versus bevacizumab and temozolomide followed by concomitant chemoradiotherapy in newly diagnosed glioblastoma multiforme: A randomized phase II study. Acta Oncol. 2014; 53:939-44. https://doi.org /10.3109/0284186x.2013.879607.

31. Reynes G, Balana C, Gallego O, Iglesias L, Perez P, Garcia JL. A phase I study of irinotecan in combination with metronomic temozolomide in patients with recurrent glioblastoma. Anticancer Drugs. 2014; 25:717-22. https:// doi.org/10.1097/cad.0000000000000059.

32. Moller S, Grunnet K, Hansen S, Schultz H, Holmberg M, Sorensen M, Poulsen HS, Lassen U. A phase II trial with bevacizumab and irinotecan for patients with primary brain tumors and progression after standard therapy. Acta Oncol. 2012; 51:797-804. https://doi.org/10.3109/02841 86x.2012.681063.

33. Reardon DA, Desjardins A, Peters KB, Gururangan S, Sampson JH, McLendon RE, Herndon JE 2nd, Bulusu A, Threatt S, Friedman AH, Vredenburgh JJ, Friedman HS. Phase II study of carboplatin, irinotecan, and bevacizumab for bevacizumab naive, recurrent glioblastoma. J
Neurooncol. 2012; 107:155-64. https://doi.org/10.1007/ s11060-011-0722-2.

34. Leonard A, Wolff JE. Etoposide improves survival in high-grade glioma: a meta-analysis. Anticancer Res. 2013; 33:3307-15.

35. Sato A, Klaunberg B, Tolwani R. In vivo bioluminescence imaging. Comp Med. 2004; 54:631-4.

36. Houghton PJ, Stewart CF, Cheshire PJ, Richmond LB, Kirstein MN, Poquette CA, Tan M, Friedman HS, Brent TP. Antitumor activity of temozolomide combined with irinotecan is partly independent of O6-methylguanineDNA methyltransferase and mismatch repair phenotypes in xenograft models. Clin Cancer Res. 2000; 6:4110-8.

37. Peters JL, Sutton AJ, Jones DR, Abrams KR, Rushton L. Contour-enhanced meta-analysis funnel plots help distinguish publication bias from other causes of asymmetry. J Clin Epidemiol. 2008; 61:991-6. https://doi. org/10.1016/j.jclinepi.2007.11.010.

38. Walker E, Hernandez AV, Kattan MW. Meta-analysis: Its strengths and limitations. Cleve Clin J Med. 2008; 75:431-9.

39. Viechtbauer W. Accounting for Heterogeneity via RandomEffects Models and Moderator Analyses in Meta-Analysis. Zeitschrift für Psychologie / Journal of Psychology. 2007; 215:104-21. https://doi.org/10.1027/0044-3409.215.2.104.

40. Macleod MR, Lawson McLean A, Kyriakopoulou A, Serghiou S, de Wilde A, Sherratt N, Hirst T, Hemblade R, Bahor Z, Nunes-Fonseca C, Potluru A, Thomson A, Baginskaite $\mathrm{J}$, et al. Risk of Bias in Reports of In Vivo Research: A Focus for Improvement. PLoS Biol. 2015; 13: e1002273. https://doi.org/10.1371/journal.pbio.1002273.

41. Easterbrook PJ, Berlin JA, Gopalan R, Matthews DR. Publication bias in clinical research. Lancet. 1991; 337:86772. https://doi.org/10.1016/0140-6736(91)90201-Y.

42. Chen L, Zhang Y, Yang J, Hagan JP, Li M. Vertebrate animal models of glioma: understanding the mechanisms and developing new therapies. Biochim Biophys Acta. 2013; 1836:158-65. https://doi.org/10.1016/j.bbcan.2013.04.003.

43. Jellinger K. Glioblastoma multiforme: Morphology and biology. Acta Neurochirurgica. 1978; 42:5-32. https://doi. org/10.1007/bf01406628.

44. Allen M, Bjerke M, Edlund H, Nelander S, Westermark B. Origin of the U87MG glioma cell line: Good news and bad news. Science Translational Medicine. 2016; 8: 354re3-re3. https://doi.org/10.1126/scitranslmed.aaf6853.

45. de Vries RB, Hooijmans CR, Tillema A, Leenaars M, Ritskes-Hoitinga M. A search filter for increasing the retrieval of animal studies in Embase. Laboratory Animals. 2011; 45:268-70. https://doi.org/10.1258/la.2011.011056.

46. Hooijmans CR, Tillema A, Leenaars M, Ritskes-Hoitinga M. Enhancing search efficiency by means of a search filter for finding all studies on animal experimentation in PubMed. Laboratory Animals. 2010; 44:170-5. https://doi. org/10.1258/la.2010.009117. 
47. Vesterinen HM, Sena ES, Egan KJ, Hirst TC, Churolov L, Currie GL, Antonic A, Howells DW, Macleod MR. Meta-analysis of data from animal studies: a practical guide. J Neurosci Methods. 2014; 221:92-102. https://doi. org/10.1016/j.jneumeth.2013.09.010.

48. Hedges LV, Gurevitch J, Curtis PS. The Meta-Analysis of Response Ratios in Experimental Ecology. Ecology. 1999; 80:1150-6. https://doi.org/10.2307/177062.

49. Duval S, Tweedie R. Trim and fill: A simple funnel-plotbased method of testing and adjusting for publication bias in meta-analysis. Biometrics. 2000; 56:455-63. https://doi. org/10.1111/j.0006-341X.

50. Egger M, Davey Smith G, Schneider M, Minder C. Bias in meta-analysis detected by a simple, graphical test. Bmj. 1997; 315:629-34. https://doi.org/10.1136/ bmj.315.7109.629
51. Gordon M, Lumley T. forestplot: Advanced Forest Plot Using 'grid' Graphics. 2017.

52. Villalonga-Planells R, Coll-Mulet L, Martínez-Soler F, Castaño E, Acebes JJ, Giménez-Bonafé P, Gil J, Tortosa A. Activation of p53 by Nutlin-3a Induces Apoptosis and Cellular Senescence in Human Glioblastoma Multiforme. PLoS One. 2011; 6:e18588. https://doi.org/10.1371/journal. pone. 0018588 .

53. Leroy B, Girard L, Hollestelle A, Minna JD, Gazdar AF, Soussi T. Analysis of TP53 Mutation Status in Human Cancer Cell Lines: A Reassessment. Human mutation. 2014; 35:756-65. https://doi.org/10.1002/humu.22556.

54. Barth RF, Kaur B. Rat brain tumor models in experimental neuro-oncology: the C6, 9L, T9, RG2, F98, BT4C, RT-2 and CNS-1 gliomas. Journal of neuro-oncology. 2009; 94:299-312. https://doi.org/10.1007/s11060-009-9875-7. 